\title{
Deep inelastic scattering on an extremal Reissner-Nordström-AdS black hole. $I$.
}

\author{
Kiminad A. Mamo* and Ismail Zahed ${ }^{\dagger}$ \\ Department of Physics and Astronomy, Stony Brook University, Stony Brook, New York 11794-3800, USA
}

(Received 4 June 2019; accepted 10 October 2019; published 17 March 2020)

\begin{abstract}
We consider deep inelastic scattering (DIS) on a large nucleus described as an extremal ReissnerNordström-AdS black hole using the holographic principle. Using the R-current correlators we determine the structure functions as a function Bjorken-x, and map it on a finite but large nucleus with fixed atomic number. The R-ratios of the nuclear structure functions exhibit strong shadowing at low-X.
\end{abstract}

DOI: 10.1103/PhysRevD.101.066013

\section{INTRODUCTION}

Deep inelastic scattering (DIS) on nuclei have shown that the nuclear structure functions deviate substantially from that of the nucleon, especially at low Bjorken-x [1-4]. The depletion at low-x is usually referred to as shadowing. It is currently understood as the coherent scattering on two or more nucleons in the nucleus, as opposed to incoherent scattering on individual nucleons. In this sense, shadowing may be reminiscent of diffractive scattering in high energy hadron-on-hadron scattering.

At extremely low-x, the measured nucleon structure function shows a rapid growth of partons, primarily gluons $[5,6]$. Phenomenological arguments suggest that the growth saturates [7], a point supported by perturbative QCD arguments [8]. A central question is then: how is the growth of low-x partons in a nucleon, realized in a nucleus? Is shadowing further enhanced at low-x? Some of these important questions will be addressed and hopefully answered in the future Electron-Ion-Collider (eIC).

DIS in holography at moderate-x is different from weak coupling as it involves hadronic and not partonic constituents [9]. The large gauge coupling causes the charges to rapidly deplete their energy and momentum, making them invisible to hard probes. However, because the holographic limit enjoys approximate conformal symmetry, the structure functions and form factors exhibit various scaling laws including the parton-counting rules [10]. In contrast, DIS scattering at low-x on a nonextremal thermal black hole was argued to be partonic and fully saturated [11].

\footnotetext{
*kiminad.mamo@stonybrook.edu

ismail.zahed@stonybrook.edu
}

Published by the American Physical Society under the terms of the Creative Commons Attribution 4.0 International license. Further distribution of this work must maintain attribution to the author(s) and the published article's title, journal citation, and DOI. Funded by SCOAP.
In this paper we consider DIS scattering on a large but finite nucleus in holography using an extremal ReissnerNordström-AdS (RN-AdS) black hole [12] in the conformal limit. We use the mapping of the RN-AdS charge on the atomic number to construct the nuclear R-ratio. This point of view takes to the extreme the concept of coherent DIS scattering on a dense nucleus, and therefore should be of relevance in the shadowing or low-x region. The intermediate- $x$ and large- $x$ regions are subleading in the holographic limit as we explain below and detail elsewhere. Our arguments will be similar to those presented for the thermal nonextremal black hole in [11], with the key difference being the large but finite charge as the atomic number.

The organization of the paper is as follows: in Sec. II we briefly review the setting for the RN-AdS black hole, and specialize to the extremal case with zero temperature. In Sec. III we detail DIS scattering on the extremal black hole in leading order in the holographic limit, and give the pertinent on-shell action for the probe gauge field. In Sec. IV we explicit the holographic structure functions on nucleus as an extremal RN-AdS black hole in the conformal limit. The R-ratio is constructed and shown to display shadowing at low-x. Our conclusions are in Sec. V.

\section{RN-AdS BLACK-HOLE}

Studies of fermionic systems in the context of gravity dual theories have been carried by many [13-15]. For dense nuclei we may simplify the nucleus by treating it as cold black hole in the conformal limit, using the holographic dual construction. In this section, we briefly review the essentials of an RN-AdS black hole, and then specialize to the extremal case.

\section{A. General RN-AdS}

The effective action describing bulk RN-AdS gravity sourcing a U(1) gauge field reads 
$S=\frac{1}{2 \kappa^{2}} \int d^{5} x \sqrt{-g}(\mathbb{R}-2 \Lambda)-\frac{1}{4 e^{2}} \int d^{5} x \sqrt{-g} F^{2}$

with $\mathbb{R}$ the Ricci scalar, $\kappa^{2}=8 \pi G_{5}$ and $\Lambda=-6 / R^{2}$ are the gravitational and cosmological constant. The ensuing gravitational equation is coupled to the Maxwell equation

$$
\begin{aligned}
& R_{m n}-\frac{1}{2} g_{m n}(\mathbb{R}-\Lambda)=\kappa^{2} T_{m n} \\
& T_{m n}=g^{p q} F_{m p} F_{q n}-\frac{1}{4} g_{m n} F^{p q} F_{p q} \\
& \frac{1}{\sqrt{-g}} \partial^{m}\left(\sqrt{-g} F_{m n}\right)=0 .
\end{aligned}
$$

Since $T_{m}^{m}=0$, the space is photon filled but with the curvature of the AdS space. The RN-AdS black-hole solution to (4.7) is charged in bulk with a U(1) scalar potential

$$
A_{t}=\mu-\frac{\mathbb{Q}}{r^{2}}
$$

and a line element

$$
d s^{2}=\frac{r^{2}}{R^{2}}\left(-f d t^{2}+d \vec{x}^{2}\right)+\frac{R^{2}}{r^{2} f} d r^{2}
$$

with

$$
f=1-\frac{m R^{2}}{r^{4}}+\frac{q^{2} R^{4}}{r^{6}}
$$

provided that the electric charge $\mathbb{Q}$ and the geometrical charge $q$ satisfy

$$
\frac{q^{2} R^{2}}{\mathbb{Q}^{2}}=\frac{4}{3} \times \frac{2 \kappa^{2}}{4 e^{2}}=\frac{R^{2}}{6 \alpha} .
$$

The last equality follows from the brane-filling setup, where the parameters can be identified as

$$
2 \kappa^{2}=\frac{8 \pi^{2} R^{3}}{N_{c}^{2}} \quad 4 e^{2}=\alpha \frac{64 \pi^{2} R}{N_{c}^{2}}
$$

with $\alpha=1$ for a U(1) R-charge, and $\alpha=\frac{1}{4} \frac{N_{c}}{N_{f}}$ for a D3-D7 $\mathrm{U}(1)$ vector charge.

\section{B. Extremal RN-AdS}

The RN-AdS black hole carries two horizons $f\left(r_{ \pm}\right)=0$ with $r_{+}>r_{-}$, which are best seen by rewriting the warping factor (2.5) as

$$
f(r)=\left(1-\frac{r_{+}^{2}}{r^{2}}\right)\left(1-\frac{r_{-}^{2}}{r^{2}}\right)\left(1+\frac{r_{+}^{2}}{r^{2}}+\frac{r_{-}^{2}}{r^{2}}\right)
$$

with $m R^{2}=r_{+}^{4}+r_{-}^{4}+r_{+}^{2} r_{-}^{2}$ and $q^{2} R^{4}=r_{+}^{2} r_{-}^{2}\left(r_{+}^{2}+r_{-}^{2}\right)$, provided that the mass $m$ and the geometrical charge $q$ satisfy $q^{4} R^{4} \leq 4 m^{3} R^{2} / 27$, with $R \sqrt{m / 3} \leq r_{+}^{2} \leq R \sqrt{m}$. The temperature of the RN-ADS black hole is fixed by the standard requirement of no conical singularity in the vicinity of the outer horizon $r_{+}$

$$
T=\frac{r_{+}^{2} f^{\prime}\left(r_{+}\right)}{4 \pi R^{2}}=\frac{r_{+}}{\pi R^{2}}\left(1-\frac{\mu^{2} \pi^{2} R^{4} \gamma^{2}}{r_{+}^{2}}\right)
$$

with $\gamma^{2}=\frac{1}{12 \pi^{2} \alpha}$. Its chemical potential $\mu$ is fixed by the zero potential condition on the outer horizon $A_{t}\left(r_{+}\right)=0$ or $\mu=\mathbb{Q} / r_{+}^{2}$. With these identifications, the standard thermodynamics typical of black holes follows.

The regulated Gibbs energy $\Omega=T \Delta S$ follows from (2.1) by inserting the RN-AdS charged black hole (2.4)(2.3) and subtracting the empty thermal AdS contribution [14]. The result is

$$
\Omega=-\frac{V_{3}}{2 \kappa^{2} R^{3}}\left(\frac{r_{+}^{4}}{R^{2}}+\frac{q^{2} R^{2}}{r_{+}^{2}}\right)
$$

by trading $m=r_{+}^{4} / R^{2}+q^{2} R^{2} / r_{+}^{2}$. The entropy $s$, energy $\epsilon$, pressure $p$ densities and density $n$ follow from (2.10) through the usual grand canonical rule $[12,14]$

$$
\begin{aligned}
& s=\frac{2 \pi r_{+}^{3}}{\kappa^{2} R^{3}} \\
& \epsilon=\frac{3 m}{2 \kappa^{2} R^{3}}=3 p \\
& n=\frac{2 \mathbb{Q}}{e^{2} R^{3}} .
\end{aligned}
$$

We will mostly consider the extremal RN-AdS black hole for which $T=0$ with $r_{+}=r_{-}=\pi R^{2} \gamma \mu$. Specifically, the bulk thermodynamical quantities in (2.11) simplify

$$
\begin{aligned}
& s=\frac{2 \pi}{\sqrt{3}} \sqrt{\alpha} n \\
& \epsilon=\frac{3}{4} n \mu=3 p \\
& n=\frac{N_{c}^{2}}{96 \pi^{2} \alpha^{2}} \mu^{3} .
\end{aligned}
$$

We note that $s \sim n \sim \epsilon / \mu \sim N_{c}^{2} \mu^{3}$. The charge of the RNAdS black hole is dual to the R-charge on the boundary carried by the gluinos in the adjoint representation. What is meaningful for our identification with a cold nucleus is the energy per particle $\epsilon / n=\frac{3}{4} \mu$, which is independent of $N_{c}$. The cold entropy per particle of the RN-AdS black hole reflects on the possible entanglement entropy per particle of a cold nucleus, but this is only suggestive. With this in mind, we identify the extremal RN-AdS black hole with a 
very large but finite nucleus of volume $V_{A}=\frac{4}{3} \pi R_{A}^{3}$ with a radius $R_{A}=R_{1} A^{\frac{1}{3}}$, a number density $A / V_{A}=n$, energy density $E_{A} / V_{A}=\epsilon$, and an energy per particle $E_{A} / A=\frac{3}{4} \mu$ (conformal). For comparison, nuclear matter with small scattering lengths carries $E_{A} / A \sim \frac{3}{5} \mu$ (free massive fermions), while neutron matter with large scattering lengths carries $E_{A} / A \sim \frac{3}{4} \mu$ close to the conformal limit.

\section{DIS ON EXTREMAL RN-AdS}

We now consider DIS scattering on a RN-AdS black hole as the hologram of DIS scattering on a large nucleus at rest in the double limit of a large number of colors and strong gauge coupling. Some useful insights on standard DIS scattering on nuclei can be found in [16] to which we refer the interested reader. For completeness, we note that DIS scattering on a nucleon using holography was first addressed in [9], and on a thermal black hole in [11]. Although the thermal black hole is rather different from the extremal RN-AdS black hole, in the DIS kinematics they will share much in common as we now detail.

\section{A. Structure functions}

To probe the RN-AdS black hole in bulk, we use the U(1) R-field $\mathbf{A}_{\mu}(x)$ as the source of the fermion bilinear 4-vector current in the boundary of $\operatorname{AdS}_{5}(r=\infty)$. We first use linear response theory to compute the boundary induced current using an on-shell action. We then relate the retarded Green function to pertinent structure functions in the DIS limit. The expectation value of the fermion current is

$$
\mathbf{J}_{\mu}(x)=-i \int d^{4} y\left\langle J_{\mu}(x) J_{\nu}(y)\right\rangle_{R} \mathbf{A}^{\nu}(y)
$$

in the linear response approximation. Here $R$ refers to the retarded correlation function in the state of finite density. In Fourier space (3.1) simplifies

$$
\mathbf{J}_{\mu}(q)=G_{\mu \nu}^{R}(q) \mathbf{A}^{\nu}(-q)
$$

with the retarded Green's function

$$
G_{\mu \nu}^{R}(q)=-i \int d^{4} y e^{i q \cdot y}\left\langle J_{\mu}(y) J_{\nu}(0)\right\rangle_{R} .
$$

The rest frame of the RN-AdS black hole selects the fixed 4-vector $n^{\mu}=(1,0,0,0)$. Current conservation and covariance yields (3.3) in terms of two invariants $R_{1,2}$

$$
\begin{aligned}
G_{\mu \nu}^{R}\left(x_{A}, q^{2}\right)= & \left(\eta_{\mu \nu}-\frac{q_{\mu} q_{\nu}}{Q^{2}}\right) R_{1}\left(x_{A}, q^{2}\right) \\
& +\left(n_{\mu}-\frac{n \cdot q}{Q^{2}} q_{\mu}\right)\left(n_{\nu}-\frac{n \cdot q}{Q^{2}} q_{\nu}\right) R_{2}\left(x_{A}, q^{2}\right) .
\end{aligned}
$$

The rest frame of the black hole is the rest frame of the nucleus with fixed energy $E_{A}=\frac{3}{4} A \mu$. Since the binding energy in a nucleus is small, we also have $E_{A} \simeq A m_{N}$ and therefore $\mu \simeq \frac{4}{3} m_{N}$. In our mapping, $m_{N}$ and $\mu$ are interchangeable for estimates. A hard photon with virtual momentum $q^{\mu}=(\omega, 0,0, q)$ scattering off the nucleus in the DIS kinematics satisfies $q^{2}-\omega^{2} \equiv Q^{2} \rightarrow \infty$ with $\omega \simeq q$ and fixed Bjorken-X

$$
x_{A}=\frac{q^{2}}{-2 q \cdot\left(n E_{A}\right)} \equiv \frac{Q^{2}}{2 E_{A} \omega}=\frac{x m_{N}}{E_{A}} .
$$

Kinematically, we expect $0 \leq x_{A} \leq 1$ or equivalently $0 \leq x \leq A$. The DIS structure functions of the RN-AdS black hole will be identified from the imaginary part of the retarded response function

$$
2 \pi F_{1}=\operatorname{Im} R_{1} \quad 2 \pi F_{2}=\frac{\omega}{E_{A}} \operatorname{Im} R_{2} .
$$

\section{B. On-shell action}

To assess (3.3), we evaluate the metric perturbation induced by the R-current $J_{\mu}$ in bulk. The corresponding gravitational wave is vectorlike $A_{m}(t, \boldsymbol{x}, u)(m=\mu, u)$ and obeys Maxwell equations in $\mathrm{AdS}_{5}$ with pertinent boundary condition at the boundary $(u=0)$ and the horizon $\left(u=u_{h}\right)$. Throughout, the holographic direction is identified with $u=\left(r_{ \pm} / r\right)^{2}$. The retarded response function (3.3) is then extracted from the induced action $\mathcal{S}[A]$ as a functional of the boundary fields $A_{\mu}(t, \boldsymbol{x}, 0)$ using

$$
G_{\mu \nu}^{R}(q)=\left.\frac{\partial^{2} \mathcal{S}_{R}}{\partial A_{\mu} \partial A_{\nu}}\right|_{A_{\mu}=A_{\mu(u=0)}} .
$$

The first few leading contributions to (3.7) are illustrated in Fig. 1(a). The first and leading contribution is of order $N_{c}^{2}$. This contribution accounts for coherent scattering on the nucleus as a whole and is dominant at low-x. At intermediate- and large- $x$, the subleading correction shown in

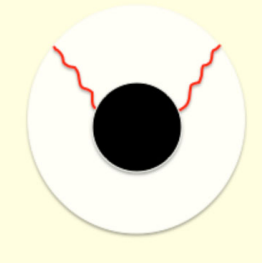

(a)

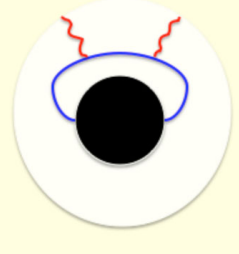

(b)
FIG. 1. Absorptive virtual-photon scattering on a nucleus as an extremal RN-AdS black hole: (a) absorptive tree contribution; (b) absorptive one-loop contribution. See text. 
Fig. 1(b) is more appropriate. The solid line refers to DIS scattering from a fermion emitted-absorbed by the black hole. It is of order $N_{c}^{0}$ and more computationally involved [17]. Suffices to say that the emission-absorption of the fermion carries information on the black hole as a cold Fermi surface [15], which can also be addressed using general arguments along with holography [18].

Equations (2.1) yield Maxwell equations in the geometry of the extremal RN-AdS black hole. In the gauge $A_{u}=0$, we dial the incoming perturbation as a plane wave with 4-momentum $q^{\mu}=(\omega, 0,0, q)$,

$$
A_{\mu}(t, x, u)=e^{-i \omega t+i q z} A_{\mu}(u)
$$

and satisfying $(i=1,2)$

$$
\begin{aligned}
\varpi A_{0}^{\prime}+k f_{0} A_{3}^{\prime} & =0 \\
A_{i}^{\prime \prime}+\frac{f_{0}^{\prime}}{f_{0}} A_{i}^{\prime}+\frac{\varpi^{2}-k^{2} f_{0}}{u f_{0}^{2}} A_{i} & =0 \\
A_{0}^{\prime \prime}-\frac{1}{u f_{0}}\left(k^{2} A_{0}+\varpi k A_{3}\right) & =0 .
\end{aligned}
$$

The prime stands for a $u$-derivative, and the dimensionless energy and momentum are

$$
\varpi \equiv \frac{\omega}{2 \pi \gamma \mu}, \quad k \equiv \frac{q}{2 \pi \gamma \mu} .
$$

For the extremal black hole, we have rewritten the metric (2.4) using $u=\left(r_{ \pm} / r\right)^{2}$,

$\mathrm{d} s^{2}=\frac{(\pi \gamma \mu R)^{2}}{u}\left(-f_{0}(u) \mathrm{d} t^{2}+\mathrm{d} x^{2}\right)+\frac{R^{2}}{4 u^{2} f_{0}(u)} \mathrm{d} u^{2}$,

with $f_{0}(u)=1-3 u^{2}+2 u^{3}$. The extremal black hole horizon $u=u_{h}=1$ solves $f_{0}\left(u_{h}\right)=0$.

There are no exact solutions to the wave equations (3.9). Following [11] we will consider the kinematical limit $k \gg K$ with $K^{2}=k^{2}-\varpi^{2}$, which corresponds to the low-x regime with $x \ll \mu / Q \sim m_{N} / Q$. The equation of motion can now be solved for $u \ll u_{h}$, with the approximate warping

$$
f_{0}(u)=1-\left(\frac{u}{\bar{\gamma}^{2}}\right)^{2}+\mathcal{O}\left(u^{3}\right)
$$

and $\bar{\gamma}^{4}=\frac{1}{3}$. In this approximation, the warping factor (3.12) for the RN-AdS black hole becomes similar to that of an ordinary thermal AdS black hole except for the differences in scaling with the chemical potential instead of the temperature. At this stage, our analysis of the longitudinal and transverse waves is similar to the one presented in [11] to which we refer for completeness.

In this regime, (3.9) are solved by sourcing the fields at the AdS boundary, e.g.,

$$
k\left(k+A_{0}+\varpi A_{3}\right)(u=0)=k^{2} \mathcal{A}_{L}(0)
$$

and similarly for the transverse wave $\mathcal{A}_{T}$, and by requiring absorptive boundary conditions for the $\mathcal{A}_{T, L}$ waves at the black-hole horizon. As a result, the induced boundary action $\mathcal{S}[A]$ develops large imaginary parts ( $c$ is Euler's constant)

$$
\begin{aligned}
\mathcal{S}_{R}= & -\frac{1}{\alpha} \frac{N_{c}^{2} \gamma^{2} \mu^{2}}{48}\left[k^{2} \mathcal{A}_{L}^{2}(0)\left(2\left(c+\ln \frac{k}{3 \bar{\gamma}^{2}}\right)-i \pi\right)\right. \\
& \left.+\frac{9 \pi}{\Gamma^{2}\left(\frac{1}{3}\right)}\left(\frac{k}{3 \bar{\gamma}^{2}}\right)^{\frac{2}{3}}\left(\frac{1}{\sqrt{3}}-i\right) \mathcal{A}_{T}^{2}(0)\right] .
\end{aligned}
$$

Modulo the overall constant in (3.14) and the rescaling by $\bar{\gamma}$, the result is in agreement with the one derived in [11] for a nonextremal thermal black hole. We now show how to use (3.14) for extracting the nuclear structure functions at low-x.

\section{HOLOGRAPHIC NUCLEAR STRUCTURE FUNCTIONS}

The holographic structure functions (3.6) are obtained by inserting (3.14) into (3.7), taking the derivatives and identifying the imaginary parts. The result is

$$
\begin{aligned}
& F_{T}\left(x_{A}, Q^{2}\right)=C_{T} \frac{\mu^{2}}{x_{A}}\left(\frac{x_{A}^{2} Q^{2}}{\mu E_{A}}\right)^{\frac{2}{3}} \\
& F_{L}\left(x_{A}, Q^{2}\right)=C_{L} \frac{E_{A}}{\mu} \frac{\mu^{2}}{x_{A}}\left(\frac{x_{A}^{2} Q^{2}}{\mu E_{A}}\right)
\end{aligned}
$$

with

$$
\begin{aligned}
C_{T} & =\frac{N_{c}^{2}}{2^{17 / 3} \pi^{2} \Gamma^{2}(1 / 3) \alpha^{5 / 3}} \\
C_{L} & =\frac{N_{c}^{2}}{1152 \pi^{4} \alpha^{2}} .
\end{aligned}
$$

For $x_{A} \ll \sqrt{\mu E_{A}} / Q$, we have $F_{L} \ll F_{T}$, which is reminiscent of the Callan-Gross relation $F_{2}=F_{L}+F_{T} \simeq 2 x_{A} F_{1}$, noted also for a thermal black hole [11]. We recall that at intermediate- $\mathrm{x}$, the structure functions on a spin- $-\frac{1}{2}$ target obey instead $F_{2}=2 F_{1}$ [9]. From (4.1) we identify the nucleus saturation line

$$
Q_{A S}\left(x_{A}\right)=\frac{\sqrt{\mu E_{A}}}{x_{A}}=\frac{\sqrt{3}}{2} \frac{\mu}{x_{A}} A^{\frac{1}{2}}
$$

for the extremal RN-AdS black hole (2.12) identified as a large nucleus (ignoring binding). The saturation momentum grows with atomic number $Q_{A S} \sim A^{\frac{1}{2}}$ since $x_{A} \sim A^{0}$. In weak coupling, simple QCD arguments for DIS suggest $Q_{A S} \approx A^{\frac{1}{3}}$. For $A=1$ it is consistent with the thermal saturation line derived in [11] with the chemical potential traded for temperature.

The chief results (4.1) are very similar to those derived in [11] aside from the kinematical identifications and the overall coefficients. This is perhaps not surprising since 
DIS scattering on partons is shifted to low-x in holography, as noted for the case of a thermal black hole in [11]. We have now confirmed this for a charged but cold black hole with a specific mapping to a cold nucleus. The thermal black hole is more appropriate for the description of a strongly coupled plasma.

\section{A. Sum rule}

The range of validity of (4.1) is limited to low-x, parametrically far from the saturation line for large $A$. To see this, it is useful to relate the leading twist in the OPE expansion of the $J J$ correlator spacelike to the moment of the structure function. For that, we note that in the deep Euclidean regime with $q^{2} \rightarrow \infty$ and $x_{A} \rightarrow \infty$, the leading twist contribution to $J J$ is the twist-2 and protected energymomentum operator $T_{\mu \nu}=\left(\eta_{\mu \nu}+4 n_{\mu} n_{\nu}\right) \epsilon / 4$. Since $R_{1,2}$ are analytic in the complex $z_{A}=1 / x_{A}$-plane minus the cuts along $\left|z_{A}-1\right| \leq 0$, we can relate the deep Euclidean region around $z_{A} \approx 0$ to the physical region along the cuts by a Cauchy transform. This procedure is standard, and the result is the sum rule involving the twist- 2 operator

$$
\begin{aligned}
\epsilon & =18 E_{A}^{2} \int_{0}^{1} d x_{A} F_{2}\left(x_{A}, Q^{2}\right) \\
& \approx 18 E_{A}^{2}\left(x_{A} F_{2}\left(x_{A}, Q^{2}\right)\right)_{x_{A} \approx \mu / A Q}
\end{aligned}
$$

The integral is dominated by the low-x region $x_{A} \approx$ $\mu / A Q \ll \mu \sqrt{A} / Q$ far from the saturation line (4.3).

\section{B. Normalization}

For a comparison with conventional structure functions in DIS on a finite nucleus, we need to address the issue of normalization. Indeed, as defined through (3.4)-(3.6), the holographic structure functions have dimensions masssquare while the standard ones are dimensionless. The reason is that in scattering off the extremal black hole the state was normalized to 1 instead of a large nucleus at rest or

$$
(2 \pi)^{3} 2 E_{A} \delta\left(\overrightarrow{0}_{p}\right) \equiv 2 E_{A} V_{A} \rightarrow(12 \pi \alpha)^{2} \frac{A^{2}}{N_{c}^{2} \mu^{2}}
$$

where the rightmost relation follows using the mapping to the extremal black hole equation of state (2.12). When inserted in (4.1) this factor yields dimensionless structure functions for DIS scattering on a cold nucleus viewed as an extremal AdS black hole. Inserting (4.5) into (4.1) and using (3.5) to trade $x_{A}$ for $x$, we obtain the properly normalized structure functions at low- $\mathrm{X}$

$$
\begin{aligned}
& F_{T}^{A}\left(x, Q^{2}\right)=\tilde{C}_{T} \frac{A}{x}\left(\frac{3 x^{2} Q^{2}}{4 m_{N}^{2}}\right)^{\frac{2}{3}} \\
& F_{L}^{A}\left(x, Q^{2}\right)=\tilde{C}_{L} \frac{3 A}{4 x}\left(\frac{3 x^{2} Q^{2}}{4 m_{N}^{2}}\right)
\end{aligned}
$$

with $\tilde{C}_{T, L} / C_{T, L}=\pi^{5}(48 \alpha)^{2} / 2 N_{c}^{2}$.

\section{R-ratio}

In holography, the structure of the nucleon at low-x is dominated by a virtual photon scattering off a spin- $\frac{1}{2}$ dilatino in bulk through a t-exchange of a Pomeron either as a surface-exchange [19], or graviton-exchange with the result [20]

$$
F_{2}^{N}(x)=\frac{C_{\Delta}}{x^{\Delta_{\mathbb{P}}}}\left(\frac{4 m_{N}^{2}}{3 Q^{2}}\right)^{\Delta-2}
$$

Here $\Delta=m R+2$ refers to the conformal dimension of the $\operatorname{spin} \frac{1}{2}$, and the Pomeron intercept is $\Delta_{\mathbb{P}}=2\left|1-\Delta^{2}\right| /$ $\sqrt{\lambda} \ll 1$, with empirically $\Delta_{\mathbb{P}} \approx 0.08$. For $\Delta=\frac{7}{2}$, the structure functions obey conformal scaling, and the corresponding hard form factors satisfy the parton-counting rules [9]. Using (4.6) and (4.7), the nuclear R-ratio follows

$$
R[x] \equiv \frac{\frac{1}{A} F_{2}^{A}}{F_{2}^{N}}=\frac{\tilde{C}_{T}}{C_{\Delta}} \frac{x^{\Delta_{\mathrm{P}}+\frac{1}{3}}}{x_{S}^{2 \Delta-\frac{8}{3}}}\left(1+\frac{3 \tilde{C}_{L}}{4 \tilde{C}_{T}}\left(\frac{x}{x_{S}}\right)^{\frac{2}{3}}\right)
$$

with $x_{S} \equiv 2 m_{N} / \sqrt{3} Q$. To compare with the experimentally measured structure functions at low-x in the shadowing region, we need to correct (4.8) by a surface contribution that is due to the finite size of the nucleus. Recall that the RN-AdS black hole occupies all of the 3-volume, which is not the case for a dense and large nucleus. This is readily done through

$$
R[x] \rightarrow R[x]+\frac{C}{A^{\frac{1}{3}}}
$$

where $C$ is a parameter that cannot be fixed by our arguments.

In Fig. 2 we show the surface corrected ratio (4.8)-(4.9) in the small-x regime $\left(x \lesssim x_{S}\right)$ as the red-dashed line, with $A=42$ and the parameters $C, C_{T, L} / C_{\Delta}$ fixed as

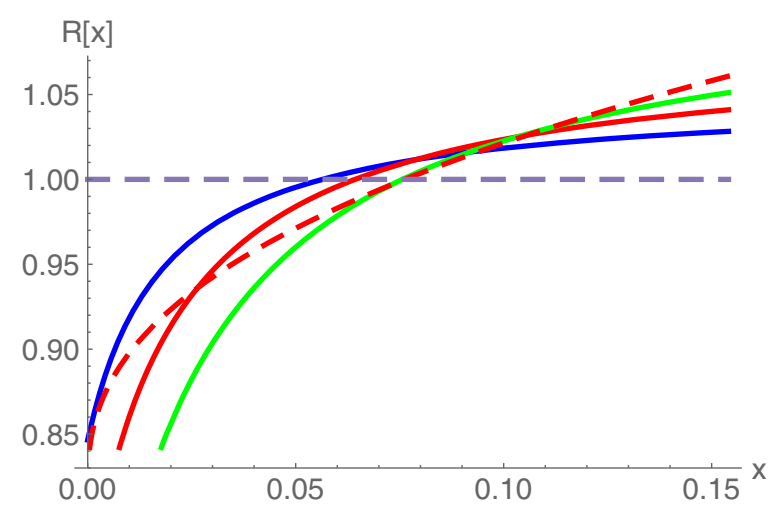

FIG. 2. Parametrized DIS data on nuclei (solid curves) vs holography (4.10) (dashed curve) in the shadowing region. See text. 


$$
0.52 x^{\frac{1}{3}+0.08}+\frac{2.85}{A^{\frac{1}{3}}}
$$

The solid curves are the HPC parametrization of the available nuclear parton distributions from [21]. The upper blue-solid line is for $A=12$, the red-middle line is for $A=42$ and the green-solid line is for $A=208$. We note that our holographic results are only justified for very low-x. They support shadowing of the low-x structure functions in DIS scattering on an RN-AdS black hole as a model for a dense nucleus.

\section{CONCLUSIONS}

We have suggested that DIS scattering on a large nucleus is analogous to DIS scattering on an extremal RN-AdS black hole in holographic QCD. In leading order the absorptive part of the scattering amplitude is dominated by coherent scattering on the bulk black hole, with structure functions that are dominant at low-x. We have mapped those results onto a finite nucleus with fixed atomic number by suitably correcting for an overall normalization. The R-ratio of the structure functions was shown to exhibit strong shadowing at low-x, an illustration of the strong depletion at low-x through absorption on the black hole. In a way DIS scattering on the RN-AdS black hole is the ultimate illustration of coherent scattering on a nucleus. The effect of Fermi motion at large-x is absent in our leading order analysis. It arises from a subleading DIS scattering on the fermions emitted and then absorbed quantum mechanically by the surface of the black hole. It will be addressed next.

\section{ACKNOWLEDGMENTS}

This work is supported by the U.S. Department of Energy under Contract No. DE-FG-88ER40388.
[1] M. R. Adams et al. (E665 Collaboration), Phys. Rev. Lett. 68, 3266 (1992); M. R. Adams et al. (Fermilab E665 Collaboration), Phys. Lett. B 287, 375 (1992); M. R. Adams et al. (E665 Collaboration), Z. Phys. C 67, 403 (1995).

[2] P. Amaudruz et al. (New Muon Collaboration), Nucl. Phys. B441, 3 (1995).

[3] J. Gomez et al., Phys. Rev. D 49, 4348 (1994).

[4] A. C. Benvenuti et al. (BCDMS Collaboration), Phys. Lett. B 189, 483 (1987).

[5] C. Adloff et al. (H1 Collaboration), Eur. Phys. J. C 13, 609 (2000); Phys. Lett. B 520, 183 (2001); Eur. Phys. J. C 21, 33 (2001).

[6] S. Chekanov et al. (ZEUS Collaboration), Eur. Phys. J. C 21, 443 (2001).

[7] K. J. Golec-Biernat and M. Wusthoff, Phys. Rev. D 59, 014017 (1998).

[8] I. Balitsky, Nucl. Phys. B463, 99 (1996); Y. V. Kovchegov, Phys. Rev. D 60, 034008 (1999).

[9] J. Polchinski and M. J. Strassler, J. High Energy Phys. 05 (2003) 012;

[10] S. J. Brodsky and G. R. Farrar, Phys. Rev. Lett. 31, 1153 (1973).

[11] Y. Hatta, E. Iancu, and A. H. Mueller, J. High Energy Phys. 01 (2008) 063.

[12] A. Chamblin, R. Emparan, C. V. Johnson, and R. C. Myers, Phys. Rev. D 60, 064018 (1999).

[13] K. Y. Kim, S. J. Sin, and I. Zahed, arXiv:hep-th/0608046; N. Horigome and Y. Tanii, J. High Energy Phys. 01 (2007) 072;

[14] S. J. Sin, J. High Energy Phys. 10 (2007) 078; X. H. Ge, Y. Matsuo, F. W. Shu, S. J. Sin, and T. Tsukioka, Prog. Theor.
Phys. 120, 833 (2008); Y. Matsuo, S. J. Sin, S. Takeuchi, T. Tsukioka, and C. M. Yoo, Nucl. Phys. B820, 593 (2009).

[15] D. T. Son and A. O. Starinets, J. High Energy Phys. 03 (2006) 052; S.S. Lee, arXiv:0809.3402; H. Liu, J. McGreevy, and D. Vegh, arXiv:0903.2477; M. Cubrovic, J. Zaanen, and K. Schalm, Science 325, 439 (2009); S. J. Sin and I. Zahed, J. High Energy Phys. 12 (2009) 015; D. T. Son, Phys. Rev. D 78, 046003 (2008); A. Adams, K. Balasubramanian, and J. McGreevy, J. High Energy Phys. 11 (2008) 059; S. S. Gubser and F. D. Rocha, Phys. Rev. D 81, 046001 (2010); A. Karch, D. T. Son, and A. O. Starinets, Phys. Rev. Lett. 102, 051602 (2009); M. Kulaxizi and A. Parnachev, Nucl. Phys. B815, 125 (2009).

[16] L. L. Frankfurt and M. I. Strikman, Phys. Rep. 160, 235 (1988).R. P. Bickerstaff and A. W. Thomas, J. Phys. G 15, 1523 (1989); M. Arneodo, Phys. Rep. 240, 301 (1994).

[17] K. A. Mamo and I. Zahed, following paper, Phys. Rev. D 101, 066014 (2020).

[18] K. A. Mamo and I. Zahed, Phys. Rev. D 100, 046015 (2019).

[19] M. Rho, S. J. Sin, and I. Zahed, Phys. Lett. B 466, 199 (1999); R. A. Janik and R. B. Peschanski, Nucl. Phys. B586, 163 (2000); E. Shuryak and I. Zahed, Ann. Phys. (Amsterdam) 396, 1 (2018).

[20] R. C. Brower, J. Polchinski, M. J. Strassler, and C. I. Tan, J. High Energy Phys. 12 (2007) 005; A. Ballon-Bayona, R. Carcassžs Quevedo, M. S. Costa, and M. Djuric, Phys. Rev. D 93, 035005 (2016);

[21] K. J. Eskola, H. Honkanen, V. J. Kolhinen, and C. A. Salgado, Phys. Lett. B 532, 222 (2002). 